\title{
Green Home: New Application for Monitoring Indoor Air Quality Using Arduino Platform
}

\author{
Ameera Ali Al-Fazari \\ Mahra Said Ahmed Al-Risi \\ Dr. Rasha Sh. AbdulWahhab
}

\author{
College of Applied Sciences, Ibri \\ College of Applied Sciences, Ibri \\ College of Applied Sciences, Ibri
}

\begin{abstract}
Air pollution is one of the most serious problems facing the atmosphere on the planet. Air pollution is defined as a collection of harmful chemicals and an organic material from factories are emitted in the atmosphere layer and causes many different diseases such as cough, eye irritation and even death. According to the World Health Organization (WHO), the number of deaths per year due to pollution from gases is about 3.5 million. The main objective of this research is to develop a real time air pollution monitoring web application able to detect indoor toxic gases titled Aircom. The proposed application has a special feature in which IoT technology is embedded in one of its units. The main purpose of using such technology is to help individual to check and get real time information about air's parameters such as Methane, Ethanol, Toluene, CO2, CO, Alcohol, Acetone, LPG, NH4, Benzene and Hexane along with the temperature, humidity and dust. Aircome will be implemented as an integrated pollution monitoring application which consist of MQ-2, MQ-3, MQ-135, MQ-9, GP2Y1010AU0F, GPS,DHT11, ESP8266 Wi-Fi, Arduino Uno board and web server. All the collected data form the suggested sensors are transmitting using Wifi technology to IoT module and in an online database. Moreover, the collected data later can be viewed using web browser which is installed in any of electronic media. The retrieved data will be displayed in the form of tables and graphs. An alert will be send by Aircom instantly in case the level of air's parameters reach above normal level. Generally speaking, Aircom will be developed by using different languages such as $\mathrm{C}++$, Arduino, Java, Java script, PHP, html and MySQL. For further verification of our proposal, we employed a quantitative study to check if what we proposed will have positive impact among different samples in the society. The outcome of the survey indicates that using such application helps to protect individuals from the bad air quality and decreases the potential health problems.
\end{abstract}

\section{Introduction}

Air pollution is one of the most serious problems facing the atmosphere on the planet. Air pollution is defined as a collection of harmful chemicals and organic materials from factories and installations in the atmosphere layer, which effects on the environment, human health and creature. According to the World Health Organization (WHO), the number of deaths per year due to pollution from gases in cooking and fuel is about 3.5 million (World Health Organization, 2018). Moreover, poor air quality in indoor environment can causes many diseases such as lung cancer, asthma and chronic obstructive.

Due to air pollution, hardware and software are provided to control indoor air quality to protect the environment. Sultanate of Oman is an example of countries that works to improve air quality. According to the Times Oman newspaper on the Sultanate's interest in this area, it explained that Sultanate has set guidelines for the community to improve indoor air quality (AL-Shihi,2019) like for example windows should be opened during cooking in order to avoid the accumulation of gases from the cooking process and burning them. In spite of the effort to motivate people and increase their awareness to alter their behavior to improve air quality but the need for a widely accessible 


\section{Journal of Student Research}

Fourth Middle East College Student Research Conference, Muscat, Sultanate of Oman

monitoring application for providing intuitive sense of air quality conditions is significant. Here we developed a new application titled Aircom for detection of harmful gases as well as dust on a user friendly web application. Indeed, Aircom is a real time air pollution monitoring web application able to detect indoor toxic gases (i.e., Methane, Ethanol, Toluene, CO2, CO, Alcohol, Acetone, LPG, NH4, Benzene and Hexane). The proposed application has a special feature in which IoT technology is embedded in one of its units. The main purpose of using such technology is to help individual to check and get real time information about air's parameters. In addition to this, Aircom is able to offer the right services all in one application that helps in the detection and improvement of indoor and outdoor air quality. Aircom consists of two main components which are the web application and hardware. The web application has many features enable users to keep track of sensed data and notifying them by email in case any high level of gases is detected. Aircom will be developed using PHP, HTML, CSS, Java Script, XAMPP. The hardware consists of multiple sensors which are MQ-2, MQ-3, MQ-135, MQ-9, GP2Y1010AU0F, DHT11 which are connected into Arduino UNO board using breadboard and jumper wires. The collected data form the suggested sensors will be send to thing speak platform via ESP8266 Wi-Fi sensor to store them later on our database and enable user to viewing them in a friendly manner at the proposed website. Therefore, data transferring can be achieved through $\mathrm{C}++$ program that needs to be uploading into the Arduino UNO using Arduino IDE software.

For further verification of our proposal, we employed a quantitative study to check if what we proposed will have positive impact among different samples in the society. A questionnaire was distributed among different samples in the society to measure the impact of using such new technology for motivating people to alter their behavior and encouraging them to use such a new technology inside the place of their residence.

The rest of this paper is presented as follows. Section 2 provides the main problem statement as well as the proposed application's objectives while section 3 explores related work. Next, all the features of the proposed application will be discussed in section 4. Section 5 explains Aircom application overview. In section 6 explains the application's results followed by a conclusion and future work in the last section.

\section{Problem Statement and Objectives}

$\mathrm{CO} 2$ and other toxic gases are one of the dangerous pollutants that cause air pollution. In Oman, there are several companies which provide devices used to detect toxic gases. But the weakness of these companies is lack to provide their services online as well as the cost of the provided devices is expensive. Moreover, checking indoor air quality for example lab users or household residents from some of potential toxic gases emission and processing capability that is obtainable on the Web for detecting toxic gases are not available in their services.

Nowadays, the technologies have been spread all around the world and used in different sectors including environment. In this research paper we will strive to resolve the entire mentioned problems by developing a low cost IOT device that will be integrated with a real time air pollution monitoring web application to detect indoor toxic gases as well as smoke and dust. The developed application has specific, measurable, achievable, realistic, timely objectives which are:

- Provide online information to protect for example lab users, householder residents and factory workers from a variety of toxic gases such as Methane, Ethanol, Toluene, CO2, CO, Alcohol, Acetone, LPG, NH4, Benzene and Hexane.

- Provide online information about particle concentration of dust in the air.

- Provide ambient room temperature and humidity information online. 


\section{Journal of Student Research}

Fourth Middle East College Student Research Conference, Muscat, Sultanate of Oman

- Notifying lab users, householder residents and factory workers in case any abnormal levels of any toxic gases are detected.

- Displaying all the sensed data at the website in form of statistics.

- Retrieved sensed data in the form of tables and graphs.

\section{Related Work}

For indoor Toxic Gas Detection System, there are many companies and devices link with applications in the Arab Gulf States and the world. The following are some examples of existing systems with their descriptions.

One of the website available in Oman is MAZOON ENVIRONMENTAL AND TECHNOLOGICAL SERVICES LLCJSRS (Al-Kalbani, 2019). This website provides environmental biotechnological solutions, environmental monitoring and testing, environmental impact assessments, environmental consulting and chemical / biological analytical services (Al-Kalbani, 2019). It provide 9 different services which are emission reporting compliance service, toxic substances monitoring services, environmental impact assessment EIA services, groundwater pollution monitoring or control, noise pollution monitoring services, soil pollution measurement or monitoring, industrial site investigation, air pollution monitoring or measurement services and oil spillage monitoring services. Beside all the above services, we found an online statistics report service is not listed as one of their services.

SGS (SGS company, 2019) is a company in UAE which offer independent services that help other businesses and company streamline their processes, reduce risks and operate in a more sustainable manner. This company provides indoor air quality test (IAQ) that is intended to make workplace more productive. The test involves regulating the ventilation climate and humidity. It also inspecting the heating and air-conditioning systems. It measures ozone, asbestos and allergens and other toxic gases. Moreover, it examines the quality of water, light intensity, noise levels and vibrations. The company is mainly doing their services with paid efforts for their employees and that will also increase the cost of their work. In addition, their website is not providing information about collected data in which the company itself and customers are not able later to check and analysis their data.

Blueair Friend is another application which works with Blueair Aware monitor device reports temperature, humidity, PM2.5, VOC's, and CO2 levels. The information is displayed in different forms such as a bar or graph. Blueair provides recommendations which help to improve air quality levels. Moreover, Blueair Aware works with Wi-Fi and sensors to measure the indoor air quality which will be send it later on to Blueair Friend app (Dina, 2018). Blueair Friend can be used in Oman but its functionality should be improve by making it capable of detecting more toxic gases that affect human in any indoor places such as schools or houses.

\section{Aircom Methodology}

Aircom application is an environmental friendly web application used to monitor and check the indoor air quality via sensors. The gases sensors will detect a variety of toxic gases such as Methane, Ethanol, Toluene, CO2, CO, Alcohol, Acetone, LPG, NH4, Benzene and Hexane. Moreover, Aircom used DHT11 and GP2Y1010AU0F sensors to check the humidity, temperature as well as dust. The sensing data from the sensors will be transmit and store in IOT platform (i.e., thing speak cloud computing services) via Wi-Fi. At the ends the data will be organize and render in our web application for the end user. Figure 1 shows the proposed Aircom architecture. Aircom has four main units which work together in order to produce the best results which are: 


\section{Journal of Student Research}

Fourth Middle East College Student Research Conference, Muscat, Sultanate of Oman

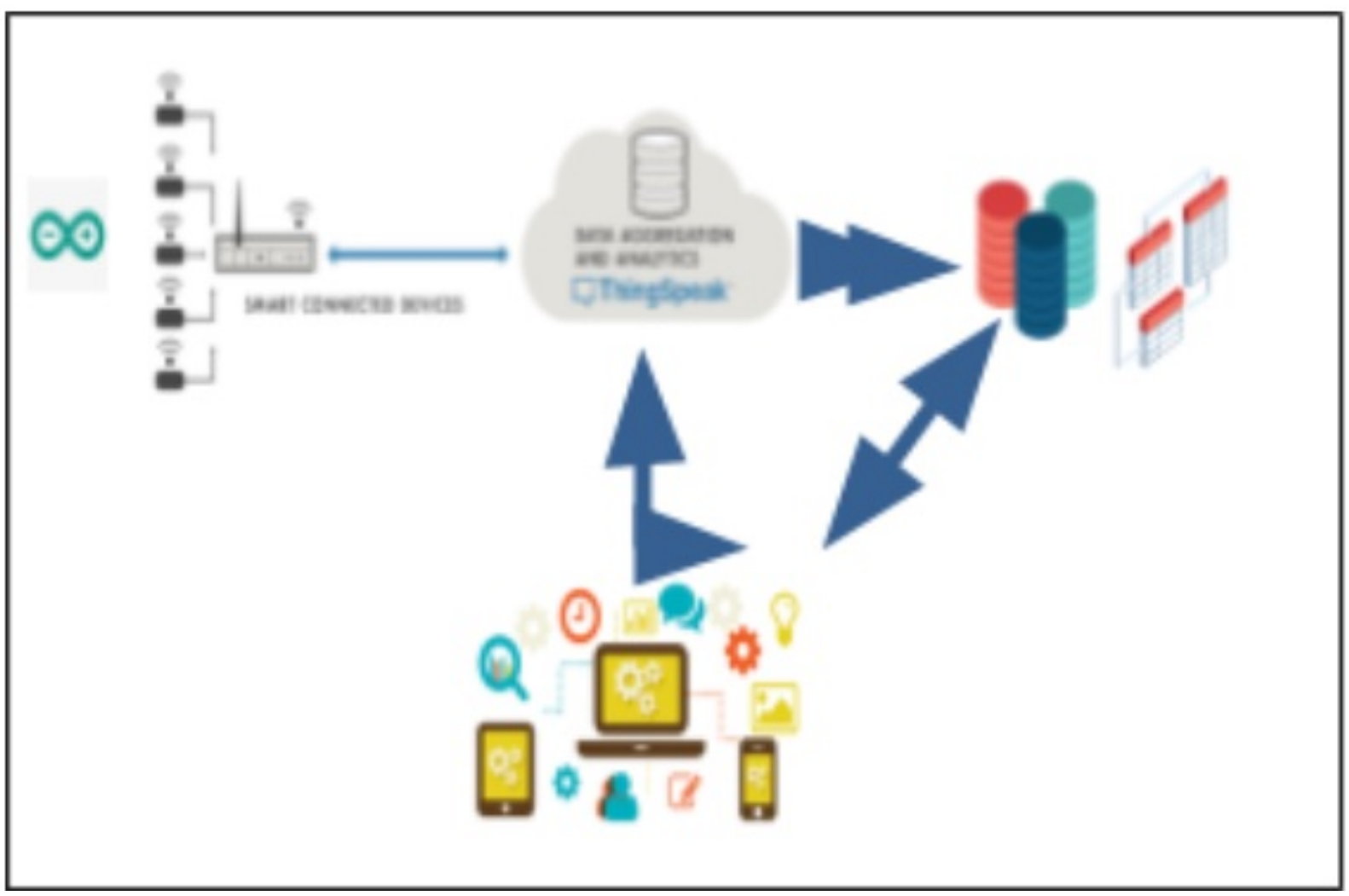

Figure 1. Aircom Architecture.

- Data Capture Unit: Aircom has multiple sensors which act as main components in our proposal which are MQ-2, MQ-3, MQ-135, MQ-9, GP2Y1010AU0F, DHT11, ESP8266 Wi-Fi, Arduino Uno board, breadboard, and jumper wires. This components will be integrated together to enable the sensors to detect any kind of gases along with dust, temperature and humidity. The sensed data will be stored to be used by the Aircom website and display the results immediately through using different display modules. Captured data will be sent through using IoT gateway to record collected data in a database using web services developed in Hypertext Preprocessor (PHP). After sensing targeted parameters, ESP8266 Wi-Fi module is responsible to forward captured data to IoT platform. The suggested sensors are programmed with Arduino IDE. Algorithm 1 illustrates the required steps of capturing and sending environmental factors to IoT platform.

\begin{tabular}{|l|l|}
\hline \multicolumn{2}{|c|}{ Algorithm 1: Pseudo code Steps of Capturing and Sending Environmental Factors. } \\
\hline 1. & Include required libraries \\
\hline 2. & Definitions \\
\hline 3. & void setup() \\
\hline 4. & \{ \\
\hline 5. & esp8266.begin(115200);//set EspSerial to 115200 \\
\hline 6. & if(Serial.find("OK")) \{ \\
\hline 7. & bool connected = connectWiFi();//Wifi connection \\
\hline 8. & if(!connected) // Check the connection is failed \\
\hline 9. & Error(); \\
\hline 10. & dht.begin();// Initialize DHT sensor. \\
\hline 11. & \} \\
\hline 12. & void loop() \\
\hline 13. & \{ \\
\hline 14. & D = dht.read(DHT11_PIN); //read data from DHT. \\
\hline 15. & GasData=GasDataRead()// Detect all Gases. \\
\hline
\end{tabular}




\section{Journal of Student Research}

Fourth Middle East College Student Research Conference, Muscat, Sultanate of Oman

\begin{tabular}{|l|l|}
\hline 16. & Dust= ReadDustData(); \\
\hline 17. & float $\mathrm{H}=$ dht.readHumidity(); // Read humidity \\
\hline 18. & float $\mathrm{F}=$ dht.readTemperature(); //Read temperature \\
\hline 19. & if (isnan(H) II isnan(F)) \{ // Check if any reads failed \\
\hline 20. & Serial.print("error"); \\
\hline 21. & return; $\}$ \\
\hline 22. & Send H,F,GasData and DustData to ThingSpeak. \\
\hline 23. & \} \\
\hline
\end{tabular}

Table 1.

Using the suggested sensor to this unit, it is possible to make Aircom able to collect and analyze required data. Circuit diagram of this unit is illustrated in figure 2 . The details of the captured data from the suggested sensors are illustrated in Table 1.

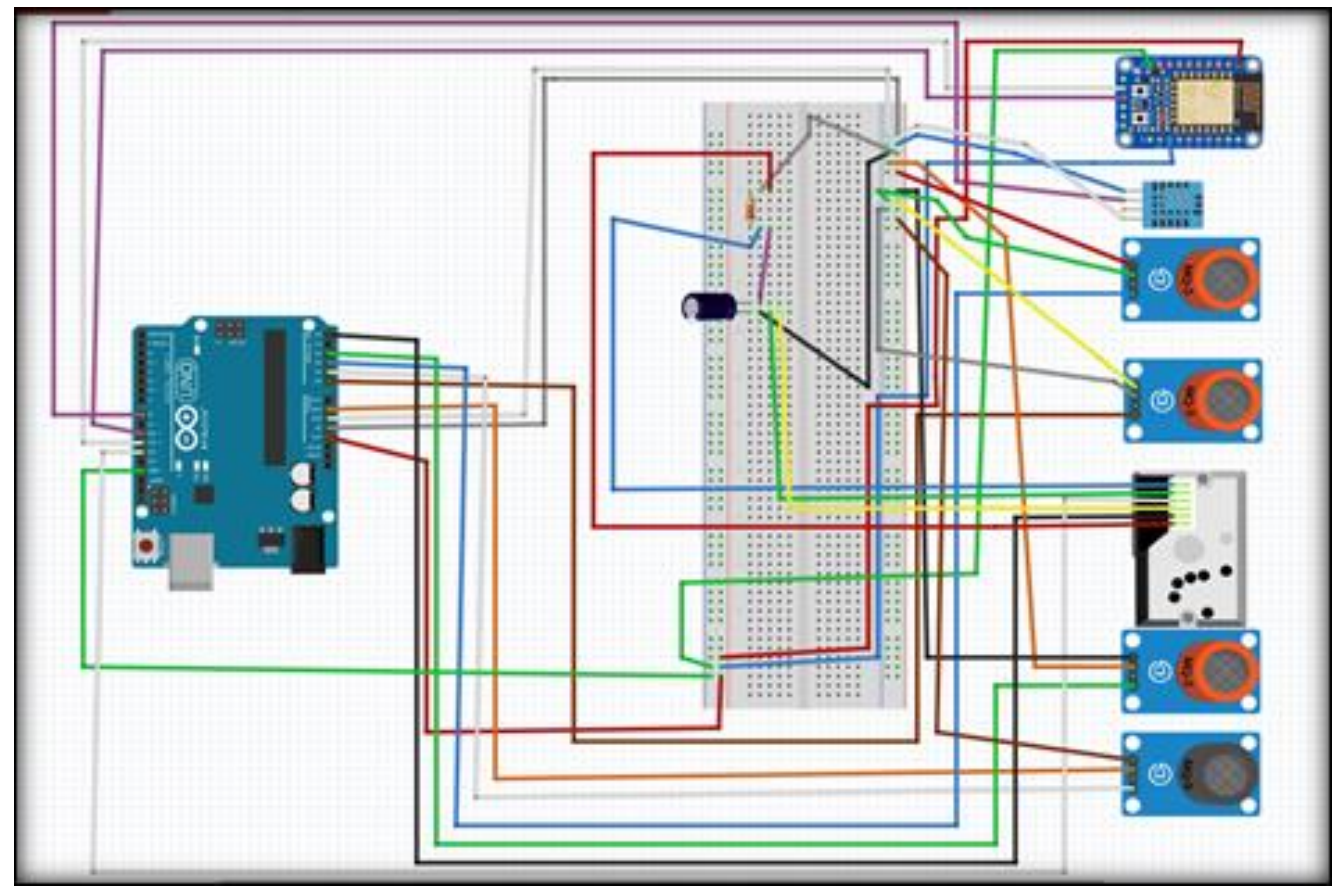

Figure 2. Circuit diagram of Aircom.

- Cloud computing unit: ESP8266 Wi-Fi will send the sensed data from the sensors into the thingSpeak cloud computing platform which will process faster and make it ready for use in our web application. The sensors feed periodically sensory data to this unit. This unit is responsible for the analysis of sensory data in an orderly and timely manner. Indeed, this unit will produce a rapid action to the lab workers or house residential for example. For instance, if the analysis result discloses abnormal rate in the quality of air, this unit will notify them by sending an alert about these conditions.

\begin{tabular}{|l|l|}
\hline Field $\#$ & Description \\
\hline Field 1 & Temperature \\
\hline Field 2 & Humidity \\
\hline Field 3 & CO \\
\hline Field 4 & CO2 \\
\hline Field 5 & Ethanol \\
\hline Field 6 & NH4 \\
\hline Field 7 & toluene \\
\hline
\end{tabular}


Journal of Student Research

Fourth Middle East College Student Research Conference, Muscat, Sultanate of Oman

\begin{tabular}{|l|l|}
\hline Field 8 & acetone \\
\hline Field 9 & dustDensity \\
\hline Field 10 & Alcohol \\
\hline Field 11 & Hexane \\
\hline Field 12 & Benzine \\
\hline Field 13 & lpg \\
\hline Field 14 & CH4 \\
\hline
\end{tabular}

Table 2. Captured Data Description.

The architecture of this unit can be depicted as two layers:

1. Information Layer: This layer is gathering, processing, and generating relevant information. This layer is connecting with IoT cloud service provider (i.e., ThingSpeak) in which an alarm or email automatically will be send in case any abnormal levels of any toxic gases are detected..

2. Decision Layer: This layer executes a simple and rapid action based on feeder information from data capture unit.

This unit launches captured information on Aircom's web site. The captured information from the sensors is displayed using varying colors and different views.

- Database unit: Aircom's web application need a database to save and make the manipulation of data easy, more efficient and fast. The database will record information like for example user's personal information, sensing data, user request information and user notification information. The basic structure of this database is made of a set of tables where information about a particular entity is represented in columns and rows.

- Displaying unit has many features and acts as interface for the user and administrator as well as the data retrieved from the sensors will be displayed in several formats such as tables or graphs.

\section{Experimental Procedures}

The potential and usability of the proposed sensor is examined in this section. Tests of sensor's components are performed by running each component through unit tests. We will test each component separately and running everything together using integration tests. We will test sensor's components of Aircom through dividing into the following functional modules to be separately tested:

- Reading DHT11 data.

- Reading data from GP2Y1010AU0F.

- Reading data form MQ-2, MQ-3, MQ-135, MQ-9.

- Connect ESP8266 Wi-Fi with Arduino Uno board.

- Sending data to the proposed IOT platform(i.e.,ThingSpeak)

DHT11 sensor is used to monitor and measure the temperature and humidity in the indoor environment. Figure 3 shows the prototype of DHT11 with Arduino Uno board. 


\section{Journal of Student Research}

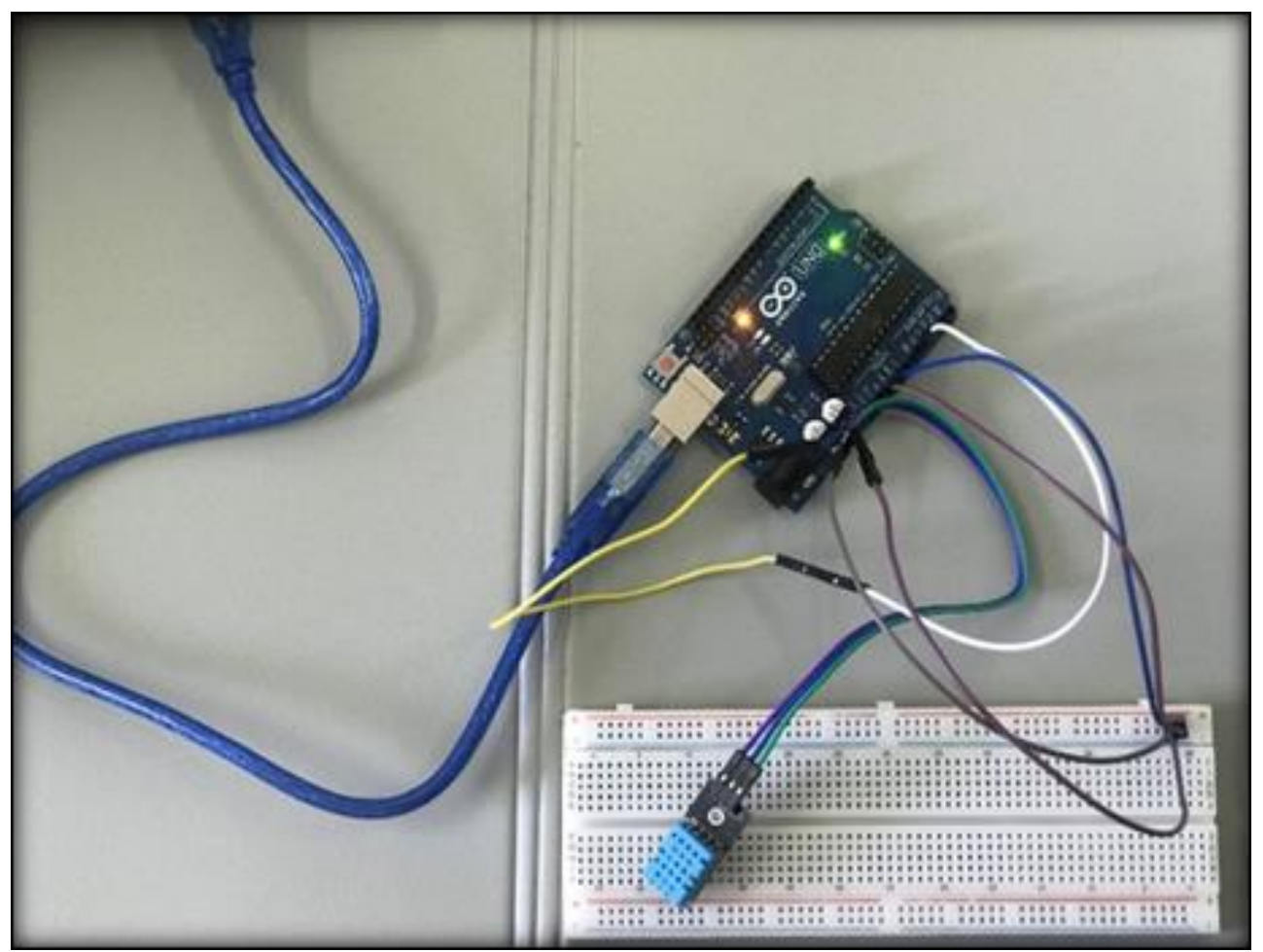

Figure 3. Prototype of DHT11 Sensor.

The serial monitor window of the sensed data by DHT11 is presented in figure 4 as we can see the sensed data are detect and represent the actual reading of temperature and humidity in the room.

Figure 4. Serial Monitor of DHT11 sensor.

GP2Y1010AU0F sensor is used to detect the dust density in the indoor environment. In this experiment, we integrate GP2Y1010AU0F sensor with DHT11 sensor. Figure 5 shows the prototype of DHT11 and GP2Y1010AU0F with Arduino Uno board.

Figure 5. Testing DHT11 Sensor and GP2Y1010AUOF.

Figure 6 illustrates the prototype of dust, MQ-2, MQ-3, MQ135, GP2Y1010AU0F, and DHT11 sensors. In this experiment, we have integrated all these sensors to check the potential and usability of the proposed sensor with Arduino Uno board. Each sensor is detecting the presence of a particular gas in the surrounding environment as well as temperature, humidity and dust value.

Figure 6. Dust, MQ-2, MQ-3, MQ135, GP2Y1010AUOF, and DHT11 Sensors.

Finally, ESP8266 Wi-Fi is integrated with the prototype of figure 6 shows the complete prototype of the proposed Aircom's sensor is illustrated in figure 7.

Figure 7. The Completed Prototype of the Proposed Aircom's Sensor

The last experiment in this section is to check the potential and usability of the proposed sensor to 
send the sensed data to Thingspeak which is IOT cloud computing platform. All the sensed data of the following figures are collected form three different location which are Suhar, Saham and Liwa. Figures 7, 8, 9 shows some values of gases that are continuously detected by the sensors having DHT11 and dust data are added besides gas sensors.

Figure 8. Value of Ethanol Gas Continuously Detected by the Sensors.

Figure 9. Value of CO Gas Continuously Detected by the Sensors.

Figure 10. Value of CH4 Gas Continuously Detected by the Sensors.

\section{Survey Results}

This paper focused also on a case study of the acceptance of people at OMAN to use this type of application at their home (i.e., Aircom) .A survey was distributed among different samples in the society and these samples were invited to contribute in this study by filling a soft copy questionnaire distributed among them online by email. The survey was consisting of five main questions. The first parts of the questionnaire were deal with personal questions such as gender and age. The total number of respondents was 20 only. As we can see from the result of figure 10 that $69.2 \%$ of samples agree that air pollution has negative impacts on the human health while $23.2 \%$ regularly disagree that. The obtain value indicates that the extent of people's knowledge on the importance of this application and its benefits on their health. The second question (see figure 11) shows that all respondents willing to obtain our idea and used such a new technology at their home. While third question (see figure 12) shows about $84.6 \%$ of respondents agreed that using such new technology will help them to detect toxic gases and that will help them to avoid different diseases such as cough, eye irritation and even death. Finally, the fourth question (see figure 13) shows that the vast majority of people have agreed to apply Aircom application. Indeed, the results showed after we analyzed them that the respondents' feedbacks were strongly positive and this encourages us to achieve our goals of issuing such new application.

Figure 11. Results of question 1.

Figure 12. Results of question 2 .

Figure 13. Results of question 3.

Figure 14. Results of question 4.

\section{The Circumstance of System Usage}

In this section, the circumstance of application usage and the workflow of the Aircom are illustrated by using the following example. Indeed, at any time the user wants to check air quality conditions or retrieving any kind of data, log into Aircom needs to be completed. Sensors are regularly capturing situational context information from the environment and processed in its 


\section{Journal of Student Research}

Fourth Middle East College Student Research Conference, Muscat, Sultanate of Oman

engine. Note that, Aircom's engine filters the data to be consumed locally and sends the rest to other Aircom's units. The circumstance of Aircom usage is described as follows:

- Users can retrieve all the collected information from the interaction with the environment, namely, displaying of temperature, gas pollution.in an easy way.

- Aircom's engine analyzes the captured data from the sensors. If the analysis result reveals abnormal rate in the quality of air, Aircom sends out the alert message to notify users about these bad conditions.

- Aircom's engine analyzes collected data to extract valuable patterns from large and complex environmental data sets.

\section{Conclusion and Future Work}

This paper proposes a smart application possessing the internet and mobile ability. The entire application consists of four major units. The proposed application can collect real-time air pollution data from a smart device. Generally speaking, the proposed application will be an essential part of the ecological environment in Oman to protect human survival from different diseases such as heart disease, strokes, and lung cancer. The main characteristics of the proposed system are:

- Aircom is an intelligent application will help to monitor air quality and enabling everyday citizens in Sultanate of Oman to check air quality.

- Aircom's units will improve decision-making and discovering solutions from the large amounts of complex data through using Data mining techniques.

- Emerging smart technology will assist in the diagnosis and monitoring polluted regions.

Finally, our vision is to introduce Aircom as a one of the most important online dynamic processing systems in Oman. Some of the additional research issues that can be investigated for future work which to expand the usability of the proposed system to monitor other regions the in the Gulf countries.

\section{Acknowledgements}

The successful to complete any work would be incomplete without constant guidance, motivate, and encourage all efforts toward success. We are grateful to our supervisor Dr. Rasha Shakir

AbdulWahhab. Her encouragement and guidance have benefited us greatly. We would like to thank College Of Applied Science in Suhar for their support and help us in collecting all necessary information and hardware needed in our project.

\section{References}

Al-Kalbani, R. (2019). Retrieved from MAZOON ENVIRONMENTAL AND TECHNOLOGICAL SERVICES LLC: https://businessgateways.com/mets

AL-Shihi, R. (2019). Improve indoor air quality at home. Times Of Oman, 6.

Dina, B. (2018, April 3). Five Best Indoor Air Quality Apps. Retrieved from sylvane Indoor Health Matters: https://www.sylvane.com/blog/five-best-indoor-air-quality-apps/

Mackenzie, J. (2016, November 1). Retrieved from Air Pollution: Everything You Need to Know: 


\section{Journal of Student Research}

Fourth Middle East College Student Research Conference, Muscat, Sultanate of Oman

https://www.nrdc.org/stories/air-pollution-everything-you-need-know

Orgnization, W. H. (2018, May 8). Household air pollution and health. Retrieved from https://www.who.int/news-room/fact-sheets/detail/household-air-pollution-and-health

SGS company. (2019). INDOOR AIR QUALITY (IAQ) .Retrieved from: https://www.sgsme.com/en/our-company/about-sgs/sgs-in-brief

Wheeler, A. (2019, February 28). Actions You Can Take to Reduce Air Pollution .Retrieved from United States Environmental Protection Agency:

https://www3.epa.gov/region1/airquality/reducepollution.html 